\section{Case Reports in Oncology}

\title{
A Case of Recurrent Ovarian Cancer and Therapy-Related Acute Myeloid Leukemia Treated with Azacitidine
}

\author{
Kenju Ando ${ }^{a}$ Akihiko Shimomura $^{a}$ Ryo Nasu $^{b}$ Misao Nakanishi ${ }^{c}$ \\ Yukino Kawamura $^{a}$ Akira Hangaishi $^{b}$ Hajime Oishi $^{c}$ Chikako Shimizu $^{a}$ \\ aDepartment of Breast and Medical Oncology, National Center for Global Health and \\ Medicine, Tokyo, Japan; bepartment of Hematology, National Center for Global Health and \\ Medicine, Tokyo, Japan; 'Department of Gynecology and Obstetrics, National Center for \\ Global Health and Medicine, Tokyo, Japan
}

\section{Keywords}

Thrombocytopenia - Therapy-related acute myeloid leukemia - Ovarian cancer - Paclitaxel ·

Carboplatin · Azacitidine

\begin{abstract}
Therapy-related myelodysplastic syndrome (tMDS) and acute myeloid leukemia (tAML) are lethal complications of chemotherapy. The incidence rates are expected to increase owing to improvements of cancer treatment. Early diagnosis of $\mathrm{TMDS} / \mathrm{AML}$ is crucial because AML progresses rapidly. Hematopoietic stem cell transplantation (HSCT) is the only current treatment to prolong survival; however, patients with tMDS/AML are more likely to be intolerable to HSCT if they have other active solid tumors. An effective treatment for patients with tMDS/ AML who are not candidates for HSCT is not established. We present a case of TAML that developed during chemotherapy for treating active ovarian cancer. The patient presented with thrombocytopenia that was initially suggested to be chemotherapy-induced thrombocytopenia. The patient was not a candidate for HSCT because of active cancer. However, she was able to receive azacitidine because her ovarian cancer responded well to chemotherapy. Pancytopenia is a common symptom of both chemotherapy-induced bone marrow suppression and tMDS/AML; thus, it may be difficult to distinguish between them at the first presentation. Given the prediction that the TMDS/AML incidence will increase as the survival of cancer patients improves, oncologists should be aware of the risks of tMDS/AML in patients with a his-
\end{abstract}


tory of cytotoxic chemotherapy. Although the indications for intensive care of tAML for patients with active solid tumors are poor, some patients might be able to receive cytotoxic treatment for tAML if the active solid tumors remain stable. Further studies focused on tMDS/ AML with active solid tumors are needed to develop an effective treatment.

(C) 2021 The Author(s).

Published by S. Karger AG, Basel

\section{Introduction}

Acute myeloid leukemia (AML) and myelodysplastic syndrome (MDS), a group of hematologic malignancies characterized by clonal proliferation of blasts, are some of the most lethal complications of chemotherapy. The WHO defines development of MDS/AML following cytotoxic therapy as therapy-related MDS (tMDS) or AML (tAML) [1]. The incidence rate of tMDS/AML has been reported as 0.62 per 100,000 person-years, which accounts for approximately $10-20 \%$ of newly diagnosed MDS/AML cases [2,3]. The number of tMDS/AML patients is expected to increase owing to improvements in cancer treatment. The National Cancer Institute's Surveillance, Epidemiology, and End Results Program, a population-based cancer registry data in the USA, reported elevated tMDS/AML risks after chemotherapy for treating solid tumors [4].

Ovarian cancer is the eighth most common cancer in women [5], and its incidence rate is expected to increase. The standard treatment is surgery followed by platinum-based chemotherapy. Without exception, chemotherapy for treating ovarian cancer is associated with a high risk of tAML/MDS $[3,6]$. The incidence rate of tMDS/AML is 5.8 cases per 10,000 personyears among patients with ovarian cancer [4].

Early diagnosis of tMDS/AML is crucial because AML progresses rapidly if untreated. However, cytopenia, a common symptom of MDS/AML, is usually observed as one of the typical adverse events of cytotoxic chemotherapy and may lead to a delay in accurate diagnosis. Moreover, treatment strategies of tAML for patients with active cancer are limited because these patients are generally not candidates for intensive treatment. Herein, we present a rare case of tAML that developed during paclitaxel- and carboplatin-based chemotherapy for treating recurrent active ovarian cancer.

\section{Case Presentation}

A 63-year-old Japanese woman with a 46-mm growing multilocular cystic mass in her left ovary was referred to the outpatient clinic of the Department of Gynecology and Obstetrics of the National Center for Global Health and Medicine in Tokyo. The patient was a healthy woman with no significant medical history. Computed tomography (CT) showed a cystic mass in the left ovary (Fig. 1a), which indicated a high possibility of malignancy. The serum CA125 level was $7.3 \mathrm{U} / \mathrm{mL}$. Subsequently, the patient underwent total abdominal hysterectomy, bilateral salpingo-oophorectomy, pelvic lymph node dissection, partial omentectomy, and partial appendectomy. Pathological examination revealed high-grade serous adenocarcinoma, and her condition was diagnosed as International Federation of Obstetrics and Gynecology stage IIA ovarian cancer. Paclitaxel and carboplatin (TC) were used as adjuvant chemotherapy at a dose of $175 \mathrm{mg} / \mathrm{m}^{2}$ and AUC 5 for 3 weeks, respectively. The patient received a total of 6 courses, and no recurrence was detected at the end of the last course (Fig. 1b).

Two years after the last chemotherapy, CT showed a recurrent tumor in her left pelvis (Fig. 1c). The serum CA125 level was $6.2 \mathrm{U} / \mathrm{mL}$. The patient received another round of TC therapy. After receiving 3 courses, she achieved a partial response (Fig. 1d). However, the following

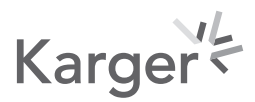



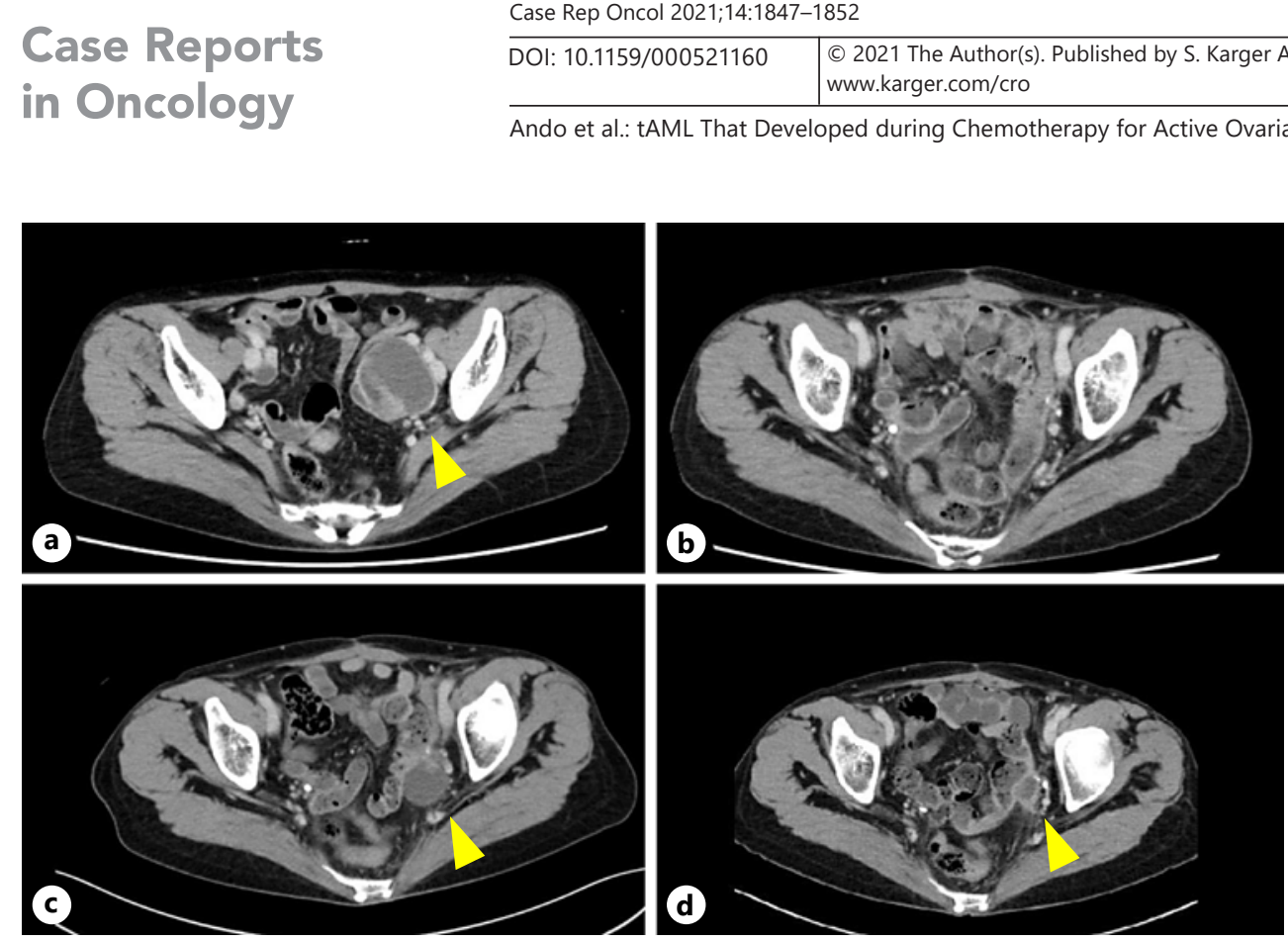

Fig. 1. CT findings. a CT on the first arrival to our hospital showing a mass in the left ovary. b CT after 6 courses of paclitaxel and carboplatin (TC) therapy following surgery showing no mass. c CT 2 years from the last chemotherapy showing a recurrent tumor in the left pelvis. $\mathbf{d}$ CT after receiving 3 courses of TC therapy showing the tumor reduced in size.

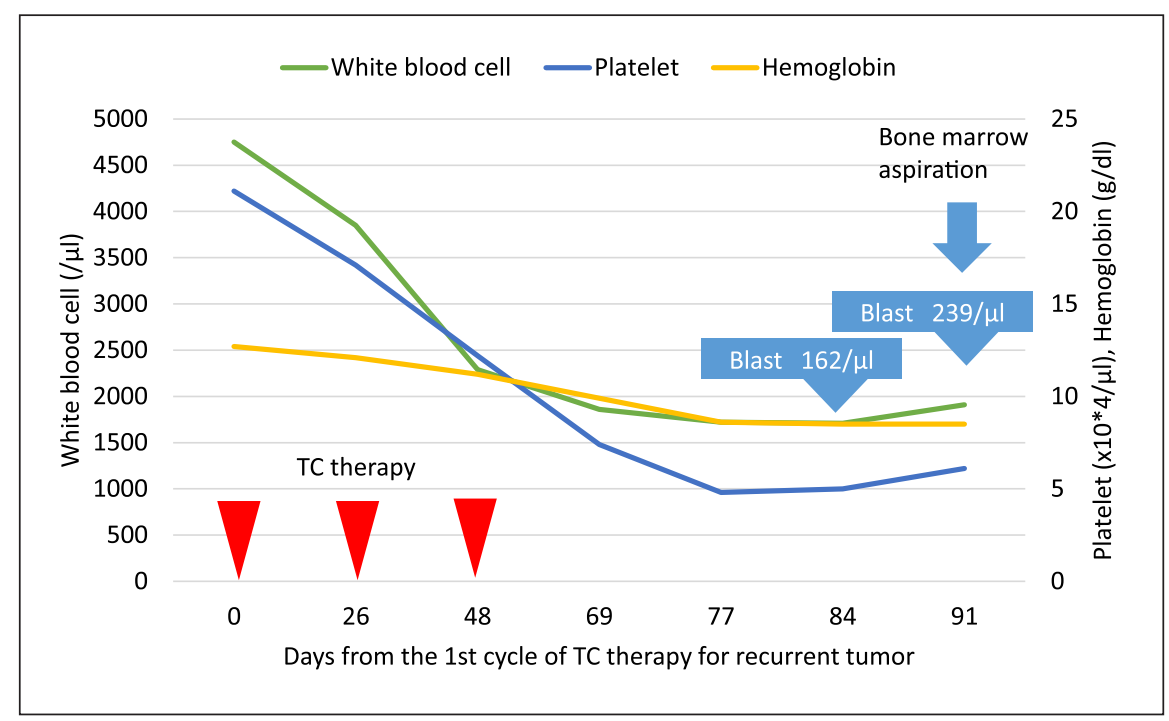

Fig. 2. Time course after the first chemotherapy for treating recurrent ovarian cancer.

chemotherapy session was postponed because of asymptomatic thrombocytopenia on day 1 of the fourth cycle of TC therapy; her platelet count was 74,000/ $\mu \mathrm{L}$ (Fig. 2). A possible diagnosis was chemotherapy-induced thrombocytopenia. Although the platelet count was expected to increase, thrombocytopenia had progressed. Additionally, anemia developed in the following week. Peripheral blood count 84 days after the first chemotherapy showed myeloblasts with Auer rods. She was immediately referred to a hematologist and underwent bone marrow aspiration, which revealed $80 \%$ myeloblasts with hypogranular dysplasia and pseudo-Pelger-Huet anomaly in granulocytes. Flow cytometry revealed the expression of CD13, CD33, CD34, and 
HLA-DR on leukemic cells. Considering dysplastic changes in the bone marrow and her history of multiple chemotherapies, the patient's condition was diagnosed as tAML. Bone marrow cytogenetic analysis showed a normal karyotype. Unfortunately, she had no indication for hematopoietic stem cell transplantation (HSCT), the only curative treatment for tAML, owing to her active ovarian cancer. She decided to cease treatment and received supportive care at her home.

Notably, CT 5 months after she ceased treatment showed that the recurrent tumor had shrunk. The serum CA125 level was $3.6 \mathrm{U} / \mathrm{mL}$. Although the patient had to receive blood transfusion 3 times a month because of progressed anemia and thrombocytopenia, her performance status was good to receive additional treatment for tAML. Six months after the diagnosis of tAML, the patient was administered azacitidine at a dose of $100 \mathrm{mg}$ for 7 days every 4 weeks. A total of 4 courses of azacitidine were administered without any severe adverse events.

\section{Discussion/Conclusion}

Generally, certain anticancer drugs, such as alkylating agents and topoisomerase II inhibitor agents, are known to have high tMDS/AML risks; the relative risks are reportedly $>10.0 \%[3,7]$. Although the associations are weak, platinum agents have been reported to cause tMDS/AML. A case-control study in North America and Europe between 1980 and 1993 reported the relationship between platinum-based chemotherapy for treating ovarian cancer and secondary leukemia; the relative risk for treatment with carboplatin was $6.5 \%$ [6]. The average latency was 4 years, and the median latency was 3.3 years. Thus, the tAML that occurred in the present case, 2 years after the first diagnosis, may have been triggered by the initial chemotherapy for treating the primary tumor.

Epithelial ovarian cancer is associated with a high risk of recurrence. The chemotherapy regimen for recurrent ovarian cancer is decided based on the time to recurrence and the platinum-free interval (PFI). Recurrent tumors with a PFI longer than 6 months are expected to be platinum-sensitive tumors [8]. Therefore, the patients described here received additional TC therapy for treating recurrent tumors. The median survival of patients with platinum-sensitive ovarian cancer has been reported to be 29 months, which is significantly better than that of patients receiving non-platinum or platinum single-agent treatments $[8,9]$. The benefit of platinum-based chemotherapy supersedes the risk of tMDS/AML in patients with ovarian cancer.

Karyotype is a well-known prognostic factor for AML. An analysis of 93 patients with tAML reported correlations between overall survival (OS) and both favorable and unfavorable cytogenetics [10]. A normal karyotype was classified as intermediate, which was observed in $14 \%$ of tAML cases. Within the group of unfavorable cytogenetics, tAML patients showed a shorter OS than de novo AML patients. In contrast, the median OS in tAML with intermediate cytogenetics was not significantly shorter than that in de novo AML (11 vs. 14 months, $p=0.31$ ).

The prognosis of tAML is poor, even if chemotherapy or radiation therapy is introduced. It has been reported that the median survival time after diagnosis of tMDS/AML is 8 months, and the 5 -year survival rate is $<10 \%$ [11]. For patients with tMDS/AML, HSCT appears to be the only current treatment to prolong survival. The median OS of patients treated with HSCT was reported to be 58.8 months, while that of patients treated with chemotherapy or radiation therapy was 12.1 months [12]. Despite the significant improvement in OS, HSCT was performed for only $17 \%$ of patients [12]. Compared with patients with de novo AML, patients with tMDS/AML are generally older and, thus, intolerable to HSCT [2].

Azacitidine is a DNA methyltransferase inhibitor that might be a preferred treatment for patients with MDS or AML who are not candidates for HSCT. Although the OS did not improve significantly, a previous phase III study has reported that azacitidine showed better OS than conventional care regimens among older patients with AML (10.4 vs. 6.5 months, hazard ratio

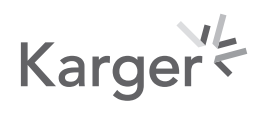


$0.85,95 \%$ confidence interval: $0.69-1.03, p=0.1009$ ) [13]. The overall response rate to azacitidine for tMDS/AML has been reported to be $38-42 \%[14,15]$. A case series of 54 patients with tMDS/AML reported that the total cycles of azacitidine closely related to the prognosis [15]. Further studies are needed to confirm the effect; however, azacitidine might be a favorable treatment for patients with tMDS/AML who are intolerable to HSCT. In general, patients with active cancer are not candidates for HSCT nor chemotherapy owing to the poor performance status. Fortunately in the case presented here, the patient was able to receive azacitidine because the recurrent ovarian tumor responded to the chemotherapy and remained stable. The patient will continue to receive further cycles of azacitidine until the disease progress.

In conclusion, we presented a case of tAML that developed during cytotoxic chemotherapy. Pancytopenia is a common symptom of both chemotherapy-induced bone marrow suppression and tMDS/AML; thus, it was difficult to distinguish between them at the first presentation. Given the prediction that the tMDS/AML incidence will increase as the survival of cancer patients improves, oncologists should be aware of the risks of tMDS/AML in patients with a history of cytotoxic chemotherapy. HSCT appears to be the only curative treatment available, although the indications for patients with active solid tumors are poor. In the case presented here, azacitidine was successfully introduced owing to the cancer's high sensitivity to chemotherapy. Further studies focused on tMDS/AML who had no indication for HSCT are needed to develop an effective treatment.

\section{Statement of Ethics}

The patient provided written informed consent for publication of the medical case and any accompanying images. This study is exempt from ethical approval by the National Center for Global Health and Medicine Institutional Review Board.

\section{Conflict of Interest Statement}

Akihiko Shimomura received grants from AstraZeneca, Chugai Pharmaceutical, DaiichiSankyo, Taiho Pharmaceutical, and Mochida Pharmaceutical, outside the submitted work; Chikako Shimizu received grants from Eli Lilly, outside the submitted work; the other authors have no conflicts of interest to declare.

\section{Funding Sources}

No funding was obtained from the private or public sector for this research.

\section{Author Contributions}

K.A. drafted the manuscript. A.S. revised the manuscript. All authors were involved in the treatment of the patient and approved the final manuscript.

\section{Data Availability Statement}

All available data including clinical details and images are included in this article.

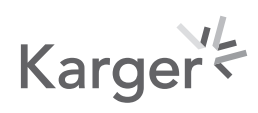




\section{Case Reports in Oncology}

\begin{tabular}{l|l}
\hline Case Rep Oncol 2021;14:1847-1852 \\
\hline DOI: 10.1159/000521160 & $\begin{array}{l}\text { @ 2021 The Author(s). Published by S. Karger AG, Basel } \\
\text { www.karger.com/cro }\end{array}$ \\
\hline
\end{tabular}

Ando et al.: tAML That Developed during Chemotherapy for Active Ovarian Cancer

\section{References}

1 Arber DA, Orazi A, Hasserjian R, Thiele J, Borowitz MJ, Le Beau MM, et al. The 2016 revision to the World Health Organization classification of myeloid neoplasms and acute leukemia. Blood. 2016;127(20):2391-405.

2 Hulegardh E, Nilsson C, Lazarevic V, Garelius H, Antunovic P, Rangert Derolf A, et al. Characterization and prognostic features of secondary acute myeloid leukemia in a population-based setting: a report from the Swedish Acute Leukemia Registry. Am J Hematol. 2015;90(3):208-14.

3 McNerney ME, Godley LA, Le Beau MM. Therapy-related myeloid neoplasms: when genetics and environment collide. Nat Rev Cancer. 2017;17(9):513-27.

4 Morton LM, Dores GM, Schonfeld SJ, Linet MS, Sigel BS, Lam CJK, et al. Association of chemotherapy for solid tumors with development of therapy-related myelodysplastic syndrome or acute myeloid leukemia in the modern era. JAMA Oncol. 2019;5(3):318-25.

5 Ferlay J, Ervik M, Lam F, Colombet M, Mery L, Piñeros M, et al. Global cancer observatory: cancer today. Lyon, France: International Agency for Research on Cancer; 2020 [cited 2021 Mar 13]. Available from: https://gco. iarc.fr/today.

6 Travis LB, Holowaty EJ, Bergfeldt K, Lynch CF, Kohler BA, Wiklund T, et al. Risk of leukemia after platinumbased chemotherapy for ovarian cancer. N Engl J Med. 1999;340(5):351-7.

7 Curtis RE, Boice JD Jr, Stovall M, Bernstein L, Greenberg RS, Flannery JT, et al. Risk of leukemia after chemotherapy and radiation treatment for breast cancer. N Engl J Med. 1992;326(26):1745-51.

8 Pfisterer J, Plante M, Vergote I, du Bois A, Hirte H, Lacave AJ, et al. Gemcitabine plus carboplatin compared with carboplatin in patients with platinum-sensitive recurrent ovarian cancer: an intergroup trial of the AGO-OVAR, the NCIC CTG, and the EORTC GCG. J Clin Oncol. 2006;24(29):4699-707.

9 Parmar MK, Ledermann JA, Colombo N, du Bois A, Delaloye JF, Kristensen GB, et al. Paclitaxel plus platinumbased chemotherapy versus conventional platinum-based chemotherapy in women with relapsed ovarian cancer: the ICON4/AGO-OVAR-2.2 trial. Lancet. 2003;361(9375):2099-106.

10 Schoch C, Kern W, Schnittger S, Hiddemann W, Haferlach T. Karyotype is an independent prognostic parameter in therapy-related acute myeloid leukemia (t-AML): an analysis of 93 patients with $\mathrm{t}-\mathrm{AML}$ in comparison to 1091 patients with de novo AML. Leukemia. 2004;18(1):120-5.

11 Smith SM, Le Beau MM, Huo D, Karrison T, Sobecks RM, Anastasi J, et al. Clinical-cytogenetic associations in 306 patients with therapy-related myelodysplasia and myeloid leukemia: the University of Chicago series. Blood. 2003;102(1):43-52.

12 Fianchi L, Pagano L, Piciocchi A, Candoni A, Gaidano G, Breccia M, et al. Characteristics and outcome of therapyrelated myeloid neoplasms: report from the Italian network on secondary leukemias. Am J Hematol. 2015; 90(5):E80-5.

13 Dombret H, Seymour JF, Butrym A, Wierzbowska A, Selleslag D, Jang JH, et al. International phase 3 study of azacitidine vs conventional care regimens in older patients with newly diagnosed AML with $>30 \%$ blasts. Blood. 2015;126(3):291-9.

14 Fianchi L, Criscuolo M, Lunghi M, Gaidano G, Breccia M, Levis A, et al. Outcome of therapy-related myeloid neoplasms treated with azacitidine. J Hematol Oncol. 2012;5:44.

15 Bally C, Thepot S, Quesnel B, Vey N, Dreyfus F, Fadlallah J, et al. Azacitidine in the treatment of therapy related myelodysplastic syndrome and acute myeloid leukemia (tMDS/AML): a report on 54 patients by the Groupe Francophone Des Myelodysplasies (GFM). Leuk Res. 2013;37(6):637-40. 